\title{
Rhein attenuates inflammation through inhibition of NF-KB and NALP3 inflammasome in vivo and in vitro
}

Hui Ge ${ }^{1, *}$

Hao Tang $2, *$

Yanbing Liang ${ }^{2}$

Jingguo $\mathrm{Wu}^{2}$

Qing Yang ${ }^{2}$

Lijin Zeng ${ }^{2}$

Zhongfu $\mathrm{Ma}^{2}$

'Department of Health Care Clinic, the First Affiliated Hospital, Sun

Yat-sen University, Guangzhou,

China; ${ }^{2}$ Department of General

Internal Medicine, the First Affiliated

Hospital, Sun Yat-sen University,

Guangzhou, China

*These authors contributed equally to this work
This article was published in the following Dove Press journal:

Drug Design, Development and Therapy

6 June 2017

Number of times this article has been viewed

\begin{abstract}
Rhein is an important component in traditional Chinese herbal medicine formulations for gastrointestinal disorders, including inflammatory bowel diseases such as ulcerative colitis. In this study, we investigated the beneficial effects of rhein in inflammation models in the transgenic zebrafish line TG (corolla eGFP), in which both macrophages and neutrophils express eGFP and RAW264.7 macrophages. We found that the tail-cutting-induced migration of immune cells was significantly reduced in transgenic zebrafish treated with rhein. In addition, the production of proinflammatory cytokines, including IL-6, IL-1 $\beta$, and tumor necrosis factor- $\alpha$, were significantly reduced in lipopolysaccharide (LPS)-induced RAW264.7 macrophages treated with rhein. Parallel to the inhibition of proinflammatory cytokines, rhein significantly reduced phosphorylation levels of NF- $\mathrm{KB}$ p 65 and inducible nitric oxide synthase, as well as COX-2 protein expression levels. Furthermore, rhein significantly reduced NALP3 and cleaved IL-1 $\beta$ expression in LPS + ATP-induced RAW264.7 macrophages. Thus, the present study demonstrates that rhein may exhibit its anti-inflammatory action via inhibition of NF- $\kappa B$ and NALP3 inflammasome pathways.
\end{abstract}

Keywords: rhein, inflammatory, zebrafish, NF-кB, iNOS, COX-2, NALP3

\section{Introduction}

Ulcerative colitis (UC) is a chronic disease of the colon and rectum. Patients with UC present with abdominal pain, diarrhea, and rectal bleeding, and these symptoms often recur and relapse. In addition to symptoms in the gastrointestinal tract, many UC patients also have psychological problems, such as depression and anxiety, which further negatively affect their quality of life. Thus, UC imposes a significant socioeconomic burden on society. Pathologically, inflammation, as evidenced by infiltration of neutrophils, macrophages, lymphocytes, and mast cells, occurs in the inner lining of the colon and rectum..$^{1-6}$ Intestinal inflammation further disrupts mucosa and submucosa, eventually leading to intestinal ulcers. The infiltration of immune cells, such as neutrophils and macrophages, has been believed to be the key factor in the local immune response in UC. Once recruited to the colon and rectum, immune cells become activated and release proinflammatory cytokines, such as interleukin (IL)-1 $\beta$, IL-6, and tumor necrosis factor (TNF)- $\alpha .{ }^{7}$ Moreover, those proinflammatory cytokines can induce activation of nuclear factor (NF)- $\mathrm{\kappa B}$, a mastering transcription factor for the regulation of inflammatory genes. Activation of NF- $\mathrm{KB}$ further induces the expression of inflammatory genes, such as COX-2 and inducible nitric oxide synthase (iNOS), which in turn recruit more proinflammatory cells and release more proinflammatory
Correspondence: Zhongfu Ma

Department of General Internal Medicine, the First Affiliated Hospital, Sun Yat-sen

University, Zhongshan Road 2,

Guangzhou 510080, China

Email mazhongfu05@sohu.com
Drug Design, Development and Therapy 2017:| | |663-167|

(c) (1) (8) ๑ 2017 Ge et al. This work is published and licensed by Dove Medical Press Limited. The full terms of this license are available at https:/www.dovepress.com/terms.php cc. hereby accept the Terms. Non-commercial uses of the work are permitted without any further permission from Dove Medical Press Limited, provided the work is properly attributed. For permision for commercial use of this work, please see paragraphs 4.2 and 5 of our Terms (htpps//www.dovepress.com/terms.php). 
cytokines, thus amplifying and prolonging inflammation in a vicious cycle. ${ }^{8}$ In addition to NF- $\kappa B$, NLRP3 inflammasome, a multiprotein complex that mediates activation of inflammatory caspases, has been recognized as an important component in UC. Physiologically, NLRP3 inflammasome is a key defense factor in the innate immune system against invading microorganisms. However, inappropriate activation of the inflammasome is linked to many inflammatory diseases. Once NLRP3 inflammasome is activated, it promotes caspase-1-mediated release of proinflammatory cytokine IL-1 $\beta$, inducing inflammation. ${ }^{9,10}$ Collectively, these inflammatory events lead to inflammation cascade effects and tissue damage in the pathological progress of UC.

The zebrafish (Danio rerio) has been extensively used to study the pathogenesis of human diseases in the field of immunology (including UC), as the innate immune system is highly conserved from mammals to zebrafish. The other advantage of zebrafish is that innate and adaptive immune responses are separated during the development of zebrafish larvae. Furthermore, zebrafish larvae are transparent, which allows real-time visualization of the inflammatory response of fluorescent cells such as fluorescent neutrophils. Additionally, zebrafish inflammatory models have successfully recapitulated the inflammatory process in UC. ${ }^{11,12}$

Rhubarb (Rheum rhabarbarum) is an important herb in formulations of traditional Chinese herbal medicine for gastrointestinal disorders including gastrointestinal hemorrhage and ulcers. Rhein (4,5-dihydroxyanthraquinone-2-carboxylic acid), the active component of rhubarb, has been shown to have multiple functions, such as antibacterial, anti-oxidant, anticancer, antiangiogenic, and anti-inflammatory effects. ${ }^{13-16}$ Given its anti-inflammatory properties, rhein has been increasingly used to treat inflammatory bowel diseases in China. However, the mechanism by which rhein attenuates inflammatory bowel diseases still remains elusive. In the present study, we examined the direct inhibitory effect of rhein on inflammation, and its anti-inflammatory mechanism in zebrafish in vivo and macrophage RAW264.7 cells in vitro.

\section{Material and methods}

\section{Chemicals and reagents}

Rhein is the main effective ingredient isolated from Rheum palmatum L. Radix et Rhizoma. It is the dried root and rhizome of R. palmatum L., R. tanguticum Maxim. ex Balf., or $R$. officinale Baill. of the family Polygonaceae, whose major active constituents are anthraquinone derivatives. The root bark of rhein belongs to the Ranunculaceae family. Rhein was purchased from the National Institute for the Control of Pharmaceutical and Biological Products (Beijing, China). The purity of the compound was $>98 \%$. It was dissolved in dimethyl sulfoxide (DMSO) and stored at $-20^{\circ} \mathrm{C}$ until use. The stock solution of rhein was further diluted in phosphate-buffered saline to achieve desired concentrations. All other reagents were obtained from Sigma-Aldrich Co. (St Louis, MO, USA) unless otherwise stated.

\section{Measurement of the toxicity of rhein}

The project was approved by the Ethics Committee of the First Affiliated Hospital of Sun Yat-sen University. Zebrafish embryos were used to evaluate the potential toxicity of rhein. Briefly, rhein was cultured in individual wells of 12 -well plates with the embryos $(n=15)$ from approximately 3-4 hours postfertilization (hpf) up to $24 \mathrm{hpf}$. The heart beat rate and survival rate of zebrafish embryos were measured at $24 \mathrm{hpf}$ and 5 days postfertilization (dpf). Heart beat rate was measured by counting and recording atrial and ventricular contractions for 3 minutes under the microscope. The data were expressed as the average heart beat rate per minute. ${ }^{17}$

\section{Preparation of inflammation-induced zebrafish model by tail cutting and application of rhein}

The transgenic zebrafish line TG (corolla: enhanced green fluorescent protein [eGFP]), which expresses eGFP in both macrophages and neutrophils, was kindly provided by the Key Laboratory of Zebrafish Modeling and Drug Screening for Human Diseases of Guangdong Higher Education Institutes, Department of Cell Biology, Southern Medical University, Guangzhou, China, and maintained as previously described. ${ }^{18,19}$ Natural pair-wise mating of fish between 3 and 12 months of age was conducted to obtain zebrafish embryos. The tail-cutting-induced inflammation assay was performed as previously described. ${ }^{20,21}$ Briefly, we first anesthetized $3 \mathrm{dpf}$ TG (corolla: eGFP) larvae with tricaine methanesulfonate solution (Sigma). With the help of a dissecting microscope, we then cut the tail of the anesthetized zebrafish larvae to remove $50 \%$ of the tail area using a blade. After tail cutting, the zebrafish larvae were placed into the wells containing different concentrations of rhein. A fluorescent inverted microscope and a couple-charged device camera were used to capture the images of zebrafish at $100 \times$ magnification during the progression of inflammation.

\section{Cell culture}

RAW264.7 mouse macrophage cells were purchased from American Type Culture Collection (Manassas, VA, USA). RAW264.7 cells were cultured in Roswell Park Memorial Institute 1640 medium supplemented with $10 \%$ heat-inactivated fetal bovine serum, glutamine, and antibiotics at $37^{\circ} \mathrm{C}$ under $5 \% \mathrm{CO}_{2}$. 


\section{Cell viability assay}

An MTT colorimetric assay was used to assess whether rhein had a cytotoxic effect on RAW264.7 macrophage cells. Cells were first seeded into 96-well plates at a density of $1 \times 10^{6} / \mathrm{mL}$ cells per well. Various concentrations of rhein $(1,5$, and $20 \mu \mathrm{M})$ were added into cultured cells. The medium was replaced by $100 \mu \mathrm{L}$ of fresh media containing MTT $(0.5 \mathrm{mg} / \mathrm{mL}) 24$ hours after rhein treatment. Three hours later, the medium was discarded and DMSO was added to dissolve the formazan crystals. The absorbance was measured at $570 \mathrm{~nm}$ on a microplate reader.

\section{Quantification of NO production}

Rhein at 1,5 , and $20 \mu \mathrm{M}$ was cultured with RAW264.7 macrophages $\left(1 \times 10^{6} / \mathrm{mL}\right)$ receiving lipopolysaccharide (LPS) for 24 hours. The Griess reaction was used to detect NO production by measuring the accumulation of nitrite, a stable end product, in the culture supernatant. ${ }^{22}$ Briefly, 100 $\mu \mathrm{L}$ of the Griess reagent was mixed with $100 \mu \mathrm{L}$ of culture supernatant from each medium in a 96-well plate, and mixed media were further incubated for 15 minutes at room temperature. A multimode microplate reader (Molecular Devices LLC, Sunnyvale, CA, USA) was used to read the spectrophotometric absorbance at $550 \mathrm{~nm}$ wavelength. A cell-free medium without nitrite served as a blank control, and sodium nitrite was used as a standard for the calculation of nitrite concentration in the medium.

\section{Measurement of proinflammatory cytokine (IL-6, IL- $\beta$, and TNF- $\alpha$ ) production}

A cytokine assay was conducted to examine the inhibitory action of rhein on proinflammatory cytokines (IL-6, IL- $\beta$, and TNF- $\alpha$ ) as previously described. ${ }^{23}$ A mouse enzymelinked immunosorbent assay (ELISA) kit (R\&D Systems, Inc., Minneapolis, MN, USA) was used to measure the proinflammatory cytokine production of supernatants from LPS- $(1 \mu \mathrm{g} / \mathrm{mL})$ treated RAW264.7 macrophages.

\section{Western immunoblot analyses}

Cell culture dishes of RAW264.7 macrophages receiving different treatments were placed on ice, washed with cold phosphate-buffered saline, and harvested with cell lysis. Bicinchoninic acid was used to determine the protein concentration of the lysate. Following addition of the sample loading buffer, protein samples were electrophoresed and then transferred to nitrocellulose membranes (Bio-Rad Laboratories Inc., Hercules, CA, USA). Nonfat milk was used to block the membrane to reduce nonspecific background. After being blocked for 1.5 hours at room temperature, the membrane was further incubated with specific primary antibodies (all obtained from Cell Signaling Technology, Danvers, MA, USA): anti-NF- $\kappa B$ p65 (1:1,000), iNOS $(1: 1,000)$, COX-2 $(1: 1,000)$, NLRP3 (1:500), and IL-1 $\beta$ (1:500).

\section{Statistics}

All data are presented as the mean \pm standard error of the mean (SEM). The Dunnett's test was used to detect the differences among test groups (Prism 4.0; GraphPad Software, Inc., La Jolla, CA, USA). A $P$-value of $<0.05$ was considered statistically significant.

\section{Results Effects of rhein on the viability of RAW264.7 cells}

The potential cytotoxic effect of rhein on RAW264.7 macrophage cells was assessed using an MTT assay. The relative MTT values were, expressed as mean \pm SEM, $100.00 \% \pm 10.21 \%$ in the control, and $98.01 \% \pm 14.56 \%$, $104.49 \% \pm 11.53 \%$, and $101.92 \% \pm 12.69 \%$ in treatment groups with rhein at 1,5 or $20 \mu \mathrm{M}$, respectively. The relative MTT values were not significantly different between the RAW264.7 macrophage cells receiving rhein at concentrations between 1 and $20 \mu \mathrm{M}$ and the control.

\section{Effect of rhein on the developmental toxicity of zebrafish embryos}

To evaluate the potential toxicity of rhein, we examined the survival rate and heart beat rate in zebrafish embryos with and without rhein. The changes in survival and heart beat rates in zebrafish embryos receiving rhein at concentrations of 1-20 $\mu \mathrm{M}$ were not significant compared with the controls. The results indicate that at tested concentrations, rhein did not induce any developmental toxicity on zebrafish embryos (Table 1). Thus, concentrations of $1-20 \mu \mathrm{M}$ of rhein were selected for subsequent experiments.

Table I Effects of rhein on the survival rate and heart beat rate of zebrafish embryos

\begin{tabular}{lll}
\hline Group & Survival rate (\%) & Heart beat rate (\%) \\
\hline Control & $99.63 \pm 9.54$ & $98.19 \pm 8.77$ \\
Rhein $(I \mu \mathrm{g} / \mathrm{mL})$ & $99.84 \pm 8.47$ & $98.73 \pm 9.61$ \\
Rhein $(5 \mu \mathrm{g} / \mathrm{mL})$ & $98.79 \pm 9.69$ & $99.54 \pm 8.84$ \\
Rhein $(20 \mu \mathrm{g} / \mathrm{mL})$ & $98.96 \pm 8.23$ & $99.72 \pm 9.79$ \\
\hline
\end{tabular}

Notes: Data are presented as mean \pm standard error of the mean. Statistical comparison of the data was performed by one-way analysis of variance followed by Newman-Keuls multiple comparison test. 
Rhein inhibited the migration of immune cells in zebrafish larvae in vivo

To investigate the anti-inflammatory action of rhein, we examined the migration of immune cells in a tail-cuttinginduced inflammation model in zebrafish. A transection of the tail in the zebrafish larvae was conducted to induce the migration of phagocytes to a site of injury. The zebrafish larvae were incubated with tested compounds for 6 hours following the transection. The mean number of fluorescent cells was $41.3 \% \pm 2.7 \%$ in tail-cutting-stimulated larvae treated with $1 \mu \mathrm{g} / \mathrm{mL}$ LPS. In contrast, the mean number of fluorescent cells were $33.4 \% \pm 3.2 \%, 25.1 \% \pm 3.4 \%$, and $15.8 \% \pm 3.1 \%$ in tail-cutting-stimulated larvae treated simultaneously with both $1 \mu \mathrm{g} / \mathrm{mL}$ LPS and different concentrations (1, 5, and $20 \mu \mathrm{M}$, respectively) of rhein (Figure 1). Collectively, treatment with rhein at 1,5 , and $20 \mu \mathrm{M}$ can dose-dependently inhibit the migration of immune cells toward the injured tail region in the larvae.

\section{Rhein reduced the production of IL-6, IL- $\beta$, and TNF- $\alpha$ in LPS-stimulated macrophages in vitro}

To determine whether rhein suppressed the production of proinflammatory cytokines such as IL-6, IL-1 $\beta$, and TNF- $\alpha$,
RAW264.7 macrophages were incubated with different concentrations of rhein $(1,5$, and $20 \mu \mathrm{M})$ in the presence or absence of LPS ( $1 \mu \mathrm{g} / \mathrm{mL})$ for 24 hours. As shown in Figure 2, compared with vehicle treatment, LPS induced a significant increase in IL-6, IL- $1 \beta$, and TNF- $\alpha$. In contrast, rhein at 5 and $20 \mu \mathrm{M}$ significantly reduced LPS-induced production of IL-6, IL-1 $\beta$, and TNF- $\alpha$.

\section{Rhein inhibited NO production in LPS-stimulated macrophages in vitro}

We further examined whether rhein could attenuate LPSinduced NO production in RAW264.7 macrophages. As shown in Figure 3, the level of NO production was significantly increased in the LPS-treated cells compared with the untreated cells. However, NO production was significantly reduced in the cells pretreated with rhein in a concentrationdependent manner.

\section{Rhein inhibited phosphorylation of NF- $\mathrm{KB}$ p65 and expression of iNOS and COX-2 in LPS-stimulated macrophages in vitro}

$\mathrm{NF}-\kappa \mathrm{B}$ is considered as a master switch in the regulation of inflammation and immunity. As a transcription factor, NF- $\kappa B$ controls an array of proinflammatory genes involved in the

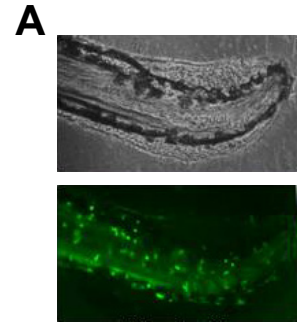

Control

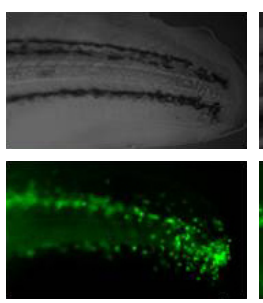

LPS $(1 \mu \mathrm{g} / \mathrm{mL})$

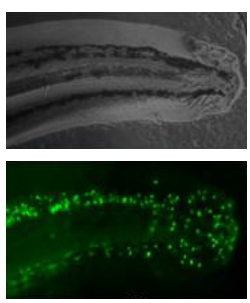

1

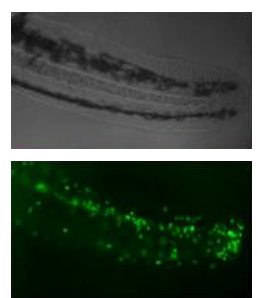

5

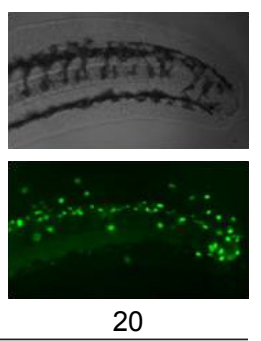

B

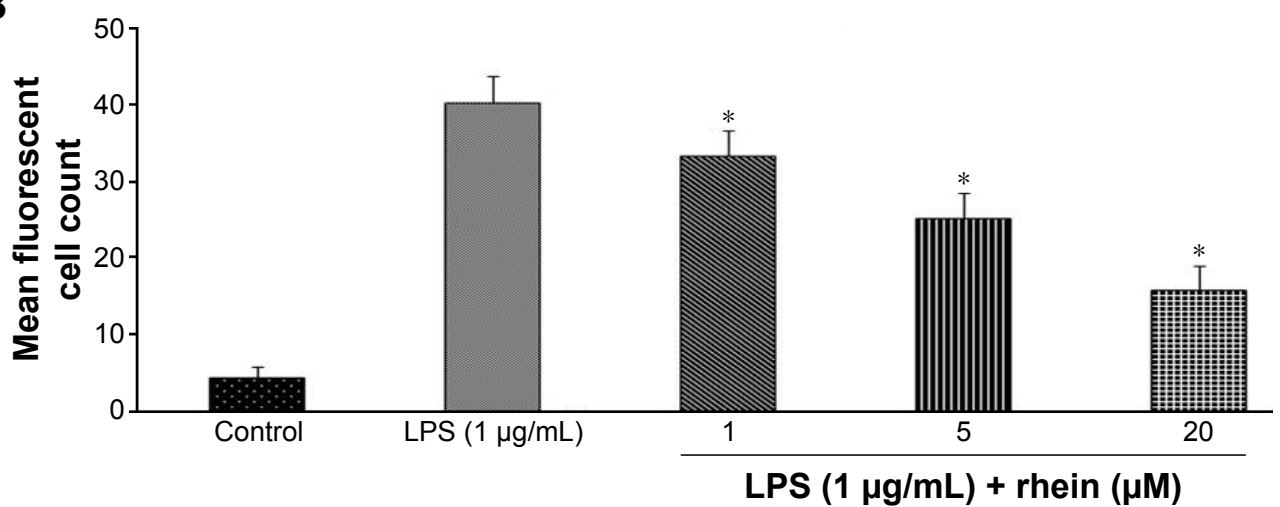

Figure I Effect of rhein on immune cell migration in tail-cutting-induced inflammation in zebrafish.

Notes: The zebrafish larvae were incubated with different concentrations of rhein following tail cutting. Representative photographs showing the fluorescence images of tail-cutting-induced immune cell migration of zebrafish larvae, 100× magnification $(\mathbf{A})$ and a bar graph showing quantitative data analysis (B). Experiments were performed in triplicate, and the data are expressed as mean \pm SEM. ( ${ }^{*} P<0.0$ I vs LPS; analysis of variance).

Abbreviations: SEM, standard error of the mean; LPS, lipopolysaccharide. 
A

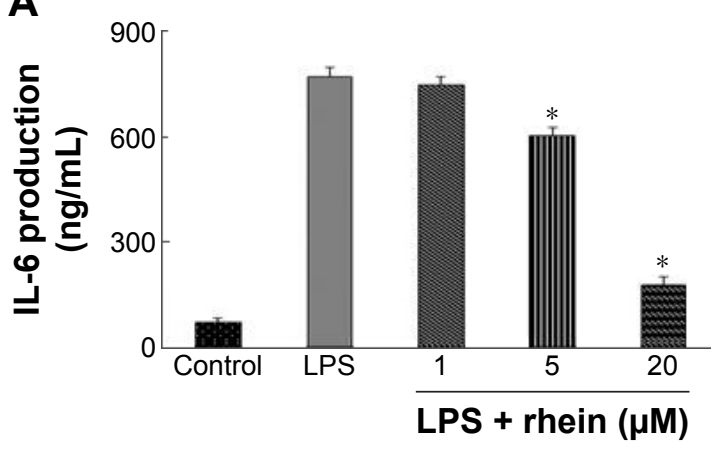

B

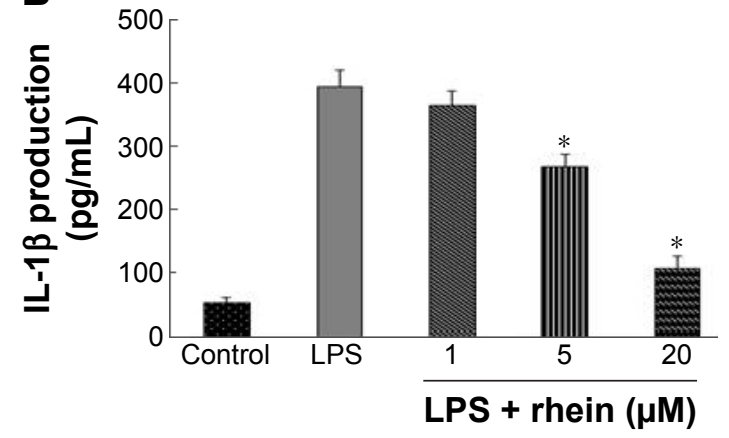

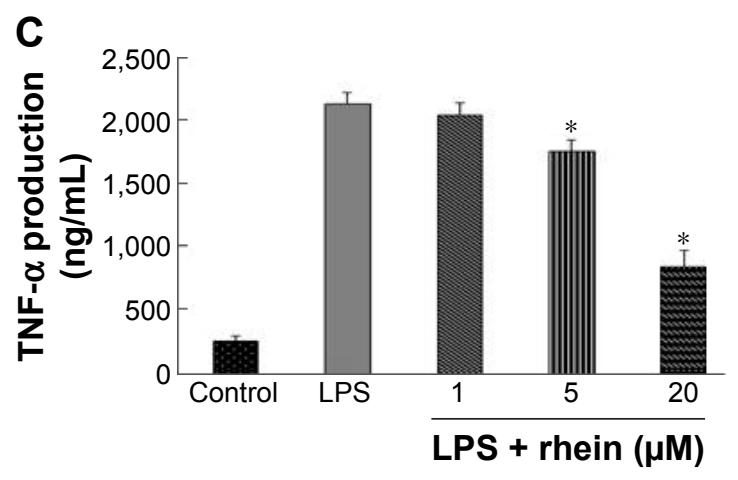

Figure 2 Effect of rhein on production of IL-6, IL-I $\beta$, and TNF- $\alpha$ in LPS-stimulated macrophages.

Notes: Macrophages were stimulated with LPS $(I \mu \mathrm{g} / \mathrm{mL})$ for 24 hours in the absence or presence of rhein (I, 5 or $20 \mu M)$. Production of IL-6, IL-I $\beta$, and TNF- $\alpha$ was assayed in the culture media using an ELISA kit (A-C). Each value represents the mean \pm SEM from three independent experiments. ( $* P<0.05$ vs $L P S$; analysis of variance). Abbreviations: ELISA, enzyme-linked immunosorbent assay; SEM, standard error of the mean; LPS, lipopolysaccharide.

inflammatory signaling cascade. NF- $\kappa \mathrm{B}$ phosphorylation has a key role in the activation of NF- $\kappa \mathrm{B}$. Phosphorylation of p65 has been shown to promote NF- $\mathrm{KB}$ activation in inflammatory conditions. Therefore, we examined whether rhein attenuated LPS-induced inflammation through inhibition of $\mathrm{NF}-\kappa \mathrm{B}$ activation. As shown in Figure 4A, treatment with $1 \mu \mathrm{g} / \mathrm{mL}$ LPS significantly increased the phosphorylation of NF- $\kappa \mathrm{B}$ p65. In contrast, pretreatment with 5 and $20 \mu \mathrm{M}$

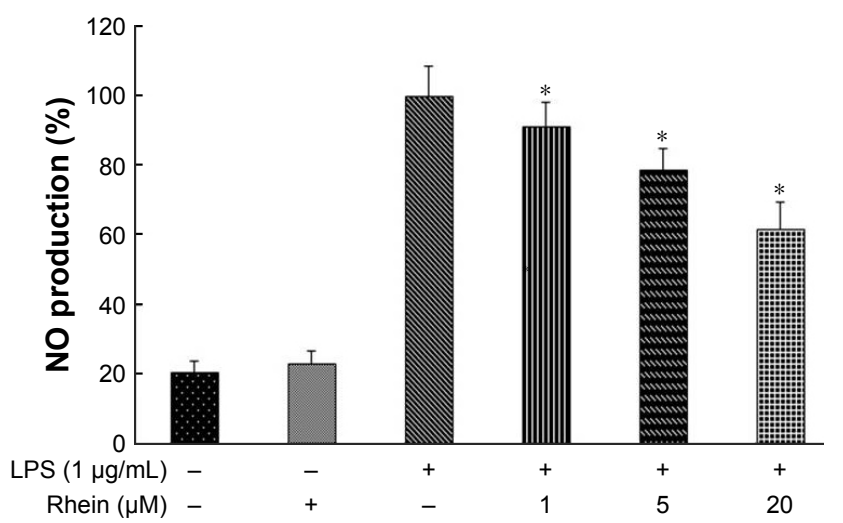

Figure 3 Effect of rhein on NO production in LPS-stimulated macrophages.

Notes: Macrophages were stimulated with LPS $(I \mu \mathrm{g} / \mathrm{mL})$ for 24 hours in the absence or presence of rhein ( 1,5 or $20 \mu \mathrm{M})$. The production of NO was assayed in the culture media. Each value represents the mean \pm SEM $(* P<0.01$ vs LPS; analysis of variance).

Abbreviation: SEM, standard error of the mean. rhein reduced phosphor-p65 levels in LPS-stimulated macrophages. iNOS and COX-2 are the major inflammatory mediators. We further examined whether rhein inhibited LPS-induced iNOS and COX-2 activation in macrophages. As shown in Figure 4B, the protein expression levels of iNOS and COX-2 were significantly increased in LPS-treated macrophages $(1 \mu \mathrm{g} / \mathrm{mL})$. In contrast, rhein significantly suppressed the protein expression levels of iNOS and COX-2 in a concentration-dependent manner.

\section{Rhein inhibited the expression levels of NLRP 3 and IL-I $\beta$ in LPS + ATP- stimulated macrophages in vitro}

NLRP3 inflammasomes regulate the maturation of IL-1 $\beta$. IL- $1 \beta$ is a potent inflammatory mediator. During the activation of IL-1 $\beta$, pro-IL-1 $\beta$ has to be processed into cleaved IL-1 $\beta$, the active form of IL-1 $\beta$. The activation of NLRP3 inflammasomes is responsible for this process. To examine the effect of rhein on NLRP3 inflammasomes, we stimulated macrophages with LPS + ATP for 24 hours. Cells were treated with or without rhein $(5$ and $20 \mu \mathrm{M})$ for 1 hour prior to stimulation. As shown in Figure 5, NLRP3 and cleaved IL-1 $\beta$ protein levels were significantly increased in LPS + ATP-stimulated macrophages. In contrast, expression levels 
A
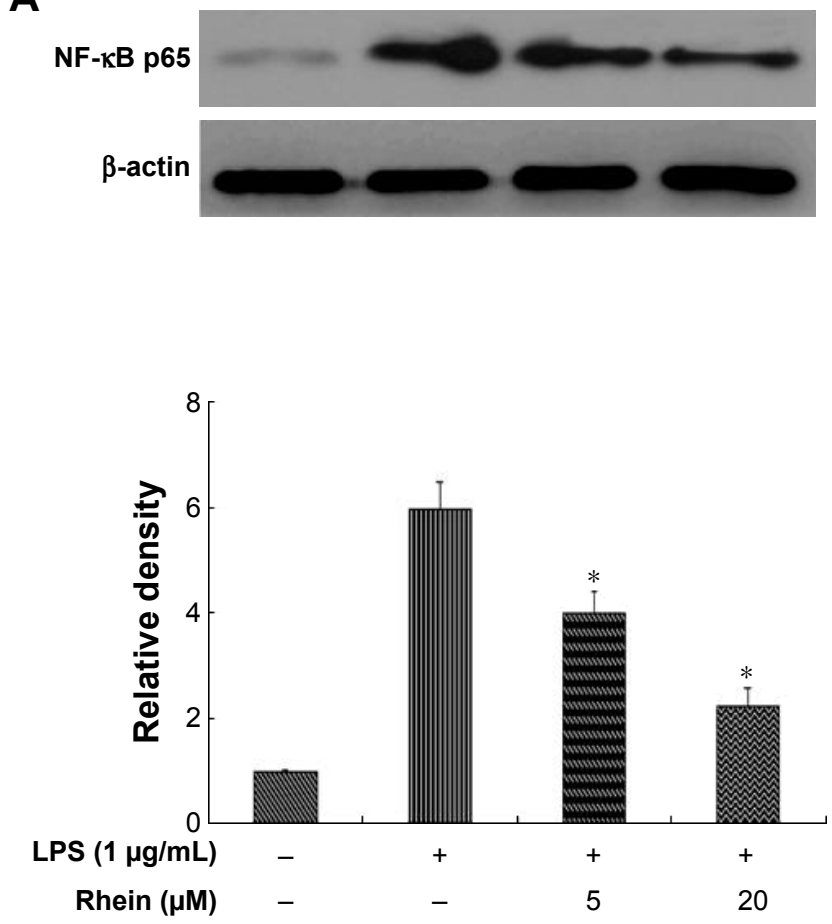

B
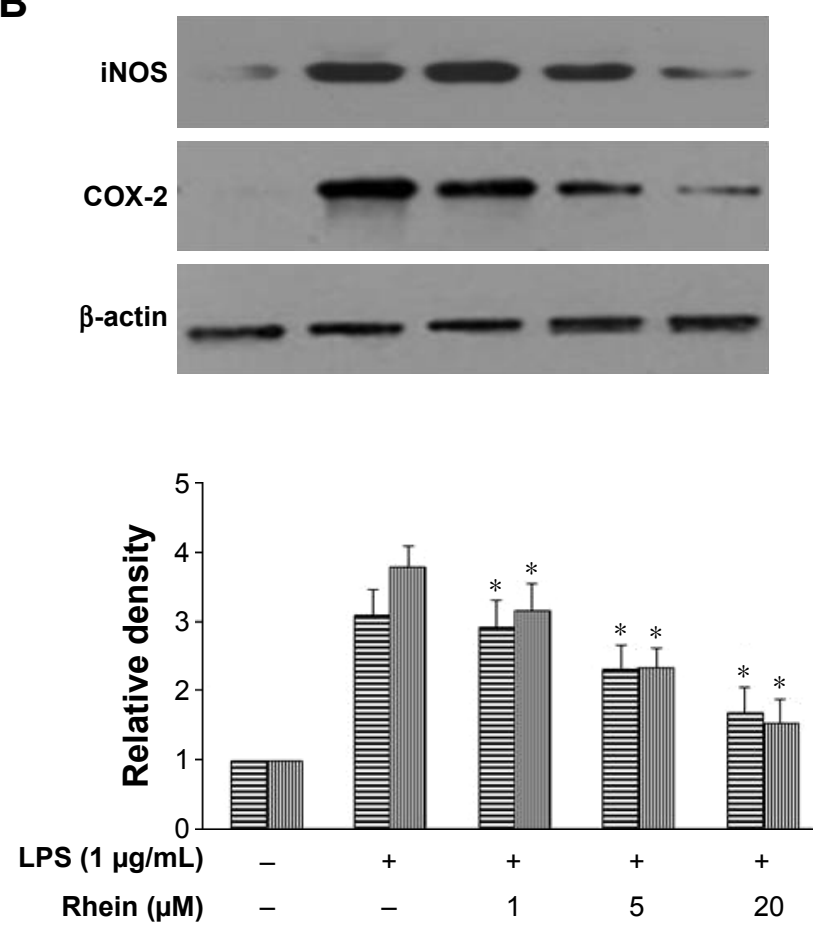

日 iNOS $\square$ COX-2

Figure 4 Effect of rhein on the expression levels of NF-KB p65, iNOS, and COX-2 in LPS-stimulated macrophages.

Notes: Representative Western blot images of NF- $\mathrm{KB}$ p65 (A), iNOS (B), and COX-2 (B) over $\beta$-actin in LPS-stimulated macrophages with or without different concentrations of rhein. The bar graph shows the quantitative analysis of densitometric data of NF- $\mathrm{KB}$, iNOS, and COX-2. Each value represents the mean \pm SEM $(* P<0.0$ I vs LPS; analysis of variance).

Abbreviations: SEM, standard error of the mean; iNOS, inducible nitric oxide synthase; NF- $\kappa B$, nuclear factor- $\kappa B$; LPS, lipopolysaccharide.

A
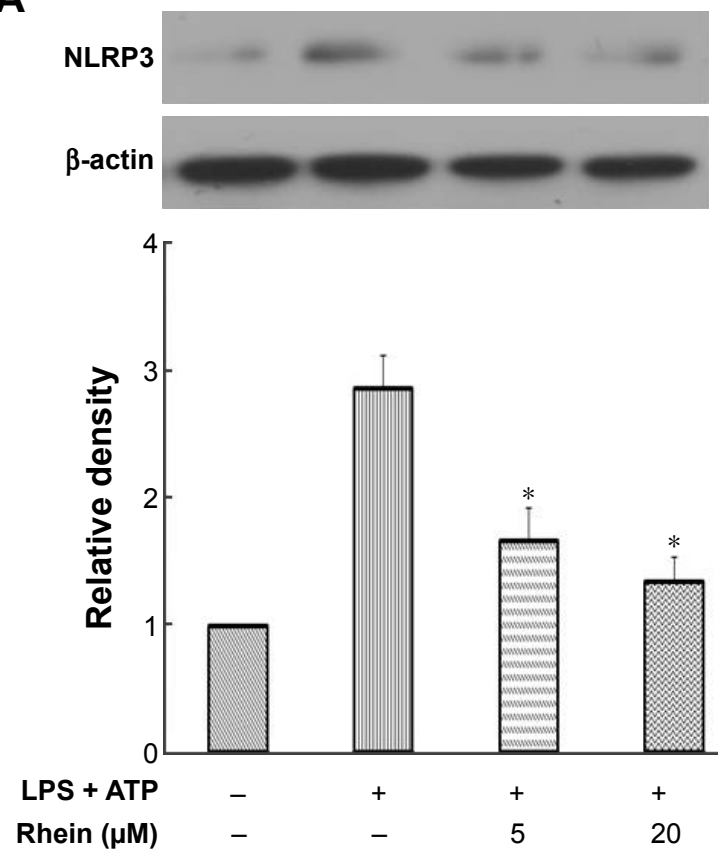

B
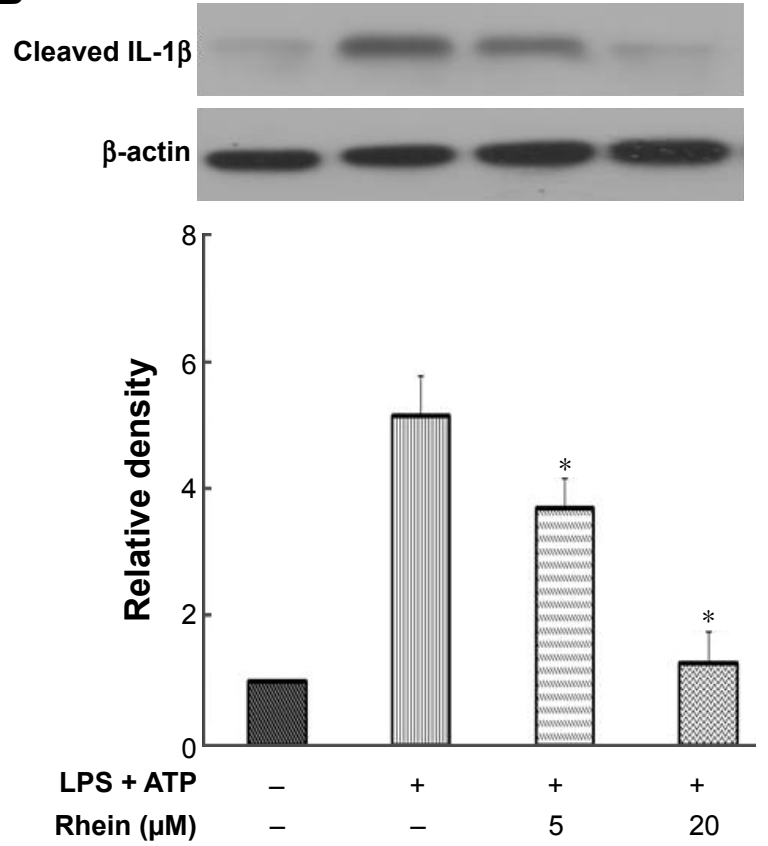

Figure 5 Effect of rhein on the expression levels of NLRP3 and IL-I $\beta$ in LPS + ATP-stimulated macrophages.

Notes: Representative Western blot images of NLRP3 (A) and IL-I $\beta$ (B) over $\beta$-actin in LPS + ATP-stimulated macrophages with or without rhein. The bar graph shows the quantitative analysis of densitometric data of NLRP3 and IL-I $\beta$. Each value represents the mean $\pm \operatorname{SEM}(* P<0.0$ I vs LPS; analysis of variance).

Abbreviations: SEM, standard error of the mean; LPS, lipopolysaccharide; IL-I $\beta$, interleukin-I $\beta$. 
of NLRP3 and IL-1 $\beta$ were significantly suppressed in macrophages receiving rhein pretreatment in a concentrationdependent manner.

\section{Discussion}

UC is a multifactorial inflammatory disease with an unknown etiology. Inflammation plays a major role in the pathophysiology of UC, while inhibition of inflammation can reduce the severity of UC. In the present study, administration of rhein reduced the tail-cutting-induced migration of immune cells in transgenic zebrafish in vivo. Rhein also reduced LPS-induced NF- $\kappa \mathrm{B}$ protein levels, iNOS and COX-2, NALP3, and cleaved IL-1 $\beta$ expression in LPS + ATPinduced RAW264.7 macrophages in vitro. This suggests that rhein may exhibit its anti-inflammatory action via inhibition of NF- $\kappa \mathrm{B}$ and NALP3 inflammasome.

The immune cells are involved in the mucosal injury during the active stage of UC. Massive infiltration of immune cells into the colorectal mucosa is a key pathological feature of UC. Once immune cells migrate into the gut, they can be primed by stimulators such as LPS of intestinal bacteria. Given that the innate and acquired immune systems are conserved between zebrafish and humans, zebrafish are good in vivo models for the assessment of anti-inflammatory effects. In the present study, we examined the anti-inflammatory action of rhein in transgenic TG (corolla: eGFP) zebrafish. In these transgenic animals, their macrophages and neutrophils are labeled with eGFP, which permitted noninvasive live imaging of the inflammation in transparent embryos and larvae.

The infiltrated immune cells are the major producers of proinflammatory cytokines in the colon. In addition, proinflammatory cytokines further promote activation and accumulation of immune cells, thus leading to a vicious cycle of inflammation. Therefore, interrupting this cycle might attenuate the inflammation and relieve the symptoms of UC. In the present study, administration of rhein significantly attenuated inflammation-induced infiltration of immune cells, such as macrophages and neutrophils in zebrafish, suggesting that rhein can directly inhibit the activity of immune cells. Several proinflammatory cytokines such as IL-6, IL-1 $1 \beta$, and TNF- $\alpha$ are believed to be the key mediators of intestinal inflammation in UC. Once those proinflammatory cytokines are released from immune cells, they further amplify inflammation, leading to cascade effects and mucosal tissue damage. IL-6, IL-1 $\beta$, and TNF- $\alpha$ are all pleiotropic cytokines that are responsible for the production of inflammatory eicosanoids, oxidative stress, and leukocyte activation and accumulation.
Increased levels of those cytokines are closely linked to clinical and histological severity of UC, whereas inhibition of proinflammatory cytokines reduces inflammatory tissue damage. Thus, those cytokines are important therapeutic targets in the treatment of UC. In the present study, rhein treatment significantly reduced proinflammatory cytokines in addition to inhibition of the migration of immune cells. Thus, the protective effects of rhein treatment may be, at least, partially, due to its inhibitory action on the release of proinflammatory cytokines from activated neutrophils and macrophages.

NO has many important physiological roles in mammals. However, it is also a free radical, and excessive NO can serve as a direct inflammatory mediator, leading to inflammation and the development of autoimmune disorders. In chronic inflammatory processes, excessive NO is mainly generated from iNOS in the immune cells. COX-2 is also involved in NO production under inflammatory conditions. In the present study, rhein significantly attenuated NO production in RAW264.7 cells. In parallel, the expression levels of iNOS and COX-2 were significantly reduced in rhein-treated RAW264.7 cells. These results suggest that rhein regulates NO production via suppression of the protein expression of iNOS and COX-2.

Physiologically, the inflammation process is tightly controlled by transcriptional factor NF- $\mathrm{B}$, a critical transcription factor for numerous proinflammatory genes, including iNOS, COX-2 and cytokines such as IL-6, IL-1 $1 \beta$, and TNF- $\alpha$. $\mathrm{NF}-\kappa \mathrm{B}$ exists as an inactive heterotrimer made up of $\mathrm{p} 50$,

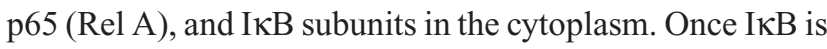
phosphorylated, NF- $\kappa \mathrm{B}$ is activated and is translocated to the nucleus, which turns on the expression of proinflammatory genes, producing proinflammatory mediators and initiating the inflammation process. The increased activation of NF- $\mathrm{KB}$ is linked to high levels of proinflammatory cytokines in human UC tissue. ${ }^{24}$ Thus, NF- $\kappa \mathrm{B}$ is regarded as a therapeutic target for UC. ${ }^{25-27} \mathrm{NF}-\kappa \mathrm{B}$ activation is tightly controlled by its p65 subunit; phosphorylation of p65 can significantly enhance the NF- $\kappa \mathrm{B}$ transcriptional response. ${ }^{28}$ In the present study, rhein significantly reduced the phosphorylation of p65 in cells treated with LPS. Collectively, these results demonstrate that rhein has a potent inhibitory action on the

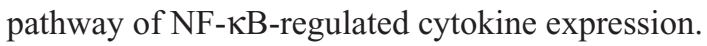

Recent studies have suggested that activation of NLRP3 inflammasome induces the production of proinflammatory cytokines in immune cells. Consistently, the expression of NLRP3 and cleaved IL- $1 \beta$ was significantly elevated in stimulated RAW264.7 cells. In contrast, pretreatment with rhein 
significantly attenuated the elevated expression of NLRP3 and cleaved IL-1 $\beta$ in stimulated RAW264.7 cells. The maturation of pro-IL-1 $\beta$ into its active form relies on activated NLRP3 inflammasome. Given that cleaved IL-1 $\beta$ is the active form of IL-1 $\beta$, the present data suggest that rhein is able to inhibit the activation of NLRP3 inflammasome.

\section{Conclusion}

In summary, treatment with rhein significantly decreased the levels of IL-6, IL-1 $\beta$, and TNF- $\alpha$, and reduced the inflammation-associated migration of immune cells. In addition, rhein reduced NO production via suppression of the protein expression of iNOS and COX-2. Furthermore, the anti-inflammatory action of rhein is, at least partially, associated with its suppression of NF- $\kappa$ B and NLRP3. Given the modulatory action of rhein on inflammatory reactions at multiple levels, the present study demonstrates that rhein may exhibit its anti-inflammatory action via inhibition of $\mathrm{NF}-\kappa \mathrm{B}$ and NALP3 inflammasome pathways. It should be noted that inflammatory bowel disease also affects other intestinal cells although macrophages have a pivotal role in inflammatory bowel disease. Thus, the present study is not specific to inflammatory bowel disease. Given that rhein has multiple functions, further studies are warranted to confirm our findings in inflammatory bowel disease at the intestinal level.

\section{Acknowledgments}

This study was supported by grants from the Science and Technology Planning Project of Guangdong Province, China (no 2012B031800291), and the Science and Technology Planning Project of Guangzhou City, China (no 201300000160).

\section{Disclosure}

The authors report no conflicts of interest in this work.

\section{References}

1. Guarner F, Malagelada JR. Role of bacteria in experimental colitis. Best Pract Res Clin Gastroenterol. 2003;17(5):793-804.

2. Kankuri E, Hämäläinen M, Hukkanen $M$, et al. Suppression of proinflammatory cytokine release by selective inhibition of inducible nitric oxide synthase in mucosal explants from patients with ulcerative colitis. Scand J Gastroenterol. 2003;38(2):186-192.

3. Stokkers PC, Hommes DW. New cytokine therapeutics for inflammatory bowel disease. Cytokine. 2004;28(4-5):167-173.

4. Cho $\mathrm{CH}$. Current roles of nitric oxide in gastrointestinal disorders. J Physiol Paris. 2001;95(1-6):253-256.

5. Danese S, Sans M, Fiocchi C. Inflammatory bowel disease: the role of environmental factors. Autoimmun Rev. 2004;3(5):394-400.

6. Zhou YX, Xia W, Yue W, Peng C, Rahman K, Zhang H. Rhein: a review of pharmacological activities. Evid Based Complement Alternat Med. 2015;2015:578107.
7. Sanchez-Munoz F, Dominguez-Lopez A, Yamamoto-Furusho JK. Role of cytokines in inflammatory bowel disease. World J Gastroenterol. 2008;14(27):4280-4288.

8. Dixit V, Mak TW. NF-kappa B signaling. Many roads lead to madrid. Cell. 2002;111(5):615-619.

9. Schroder K, Tschopp J. The inflammasomes. Cell. 2010;140(6): 821-832.

10. Schroder K, Zhou R, Tschopp J. The NLRP3 inflammasome: a sensor for metabolic danger? Science. 2010;327(5963):296-300.

11. Phelps HA, Neely MN. Evolution of the zebrafish model: from development to immunity and infectious disease. Zebrafish. 2005;2(2): $87-103$.

12. Van der Sar AM, Appelmelk BJ, Vandenbroucke-Grauls CM, Bitter W. A star with stripes: zebrafish as an infection model. Trends Microbiol. 2004;12(10):451-457.

13. Zhao YL, Zhou GD, Yang HB, et al. Rhein protects against acetaminophen-induced hepatic and renal toxicity. Food Chem Toxicol. 2011;49(8):1705-1710.

14. Irshad, Shreaz S, Manzoor N, Khan LA, Rizvi MM. Anticandidal activity of Cassia fistula and its effect on ergosterol biosynthesis. Pharm Biol. 2011;49(7):727-733.

15. Fernand VE, Losso JN, Truax RE, et al. Rhein inhibits angiogenesis and the viability of hormone-dependent and -independent cancer cells under normoxic or hypoxic conditions in vitro. Chem Biol Interact. 2011;192(3):220-232.

16. Choi RJ, Ngoc TM, Bae K, et al. Anti-inflammatory properties of anthraquinones and their relationship with the regulation of P-glycoprotein function and expression. Eur J Pharm Sci. 2013;48(1-2): 272-281.

17. Cha SH, Ko SC, Kim D, Jeon YJ. Screening of marine algae for potential tyrosinase inhibitor: those inhibitors reduced tyrosinase activity and melanin synthesis in zebrafish. J Dermatol. 2011;38(4):354-363.

18. Jin H, Sood R, Xu J, et al. Definitive hematopoietic stem/progenitor cells manifest distinct differentiation output in the zebrafish VDA and PBI. Development. 2009;136(4):647-654.

19. Li L, Yan B, Shi YQ, Zhang WQ, Wen ZL. Live imaging reveals differing roles of macrophages and neutrophils during zebrafish tail fin regeneration. J Biol Chem. 2012;287(30):25353-25360.

20. Lieschke GJ, Oates AC, Crowhurst MO, Ward AC, Layton JE. Morphologic and functional characterization of granulocytes and macrophages in embryonic and adult zebrafish. Blood. 2001;98(10): 3087-3096.

21. Hall C, Flores MV, Storm T, Crosier K, Crosier P. The zebrafish lysozyme $\mathrm{C}$ promoter drives myeloid-specific expression in transgenic fish. BMC Dev Biol. 2007;7:42.

22. Szliszka E, Skaba D, Czuba ZP, Krol W. Inhibition of inflammatory mediators by neobavaisoflavone in activated RAW264.7 macrophages. Molecules. 2011;16(5):3701-3712.

23. Cho JY, Baik KU, Jung JH, Park MH. In vitro anti-inflammatory effects of cynaropicrin, a sesquiterpene lactone, from Saussurea lappa. Eur J Pharmacol. 2000;398(3):399-407.

24. Gan HT, Chen YQ, Ouyang Q. Sulfasalazine inhibits activation of nuclear factor-kappaB in patients with ulcerative colitis. $J$ Gastroenterol Hepatol. 2005;20(7):1016-1024.

25. Karrasch T, Jobin C. NF-kappa B and the intestine: friend or foe? Inflamm Bowel Dis. 2008;14(1):114-124.

26. Perkins ND, Gilmore TD. Good cop, bad cop: the different faces of NF-kappa B. Cell Death Differ. 2006;13(5):759-772.

27. Hayden MS, Ghosh S. Signaling to NF-kappaB. Gene Dev. 2004; 18(18):2195-2224.

28. Buss H, Handschick K, Jurrmann N, et al. Cyclin-dependent kinase 6 phosphorylates NF- $\mathrm{\kappa B}$ P65 at serine 536 and contributes to the regulation of inflammatory gene expression. PLoS One. 2012;7(12):e51847. 


\section{Publish your work in this journal}

Drug Design, Development and Therapy is an international, peerreviewed open-access journal that spans the spectrum of drug design and development through to clinical applications. Clinical outcomes, patient safety, and programs for the development and effective, safe, and sustained use of medicines are the features of the journal, which has also been accepted for indexing on PubMed Central. The manuscript management system is completely online and includes a very quick and fair peer-review system, which is all easy to use. Visit http://www.dovepress.com/testimonials.php to read real quotes from published authors.

Submit your manuscript here: http://www.dovepress.com/drug-design-development-and-therapy-journal 